\title{
LA COHÉRENCE TEMPORELLE EN OPTIQUE CLASSIQUE
}

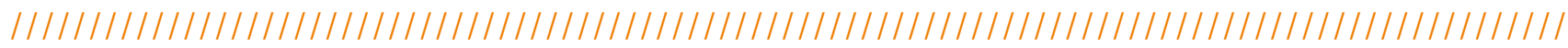

\section{Pierre CHAVEL ${ }^{1,2}$}

${ }^{1}$ Institut d'Optique Graduate School, Université Paris-Saclay, Palaiseau

${ }^{2}$ Laboratoire Hubert Curien, Université de Lyon/Université Jean-Monnet de Saint-Etienne

*pierre.chavel@institutoptique.fr
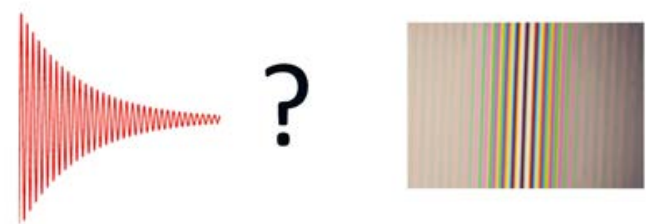

https://doi.org/10.1051/photon/202111151
Déterminante pour l'observation d'interférences, la cohérence temporelle est souvent reliée à la comparaison entre différence de marche et longueur de «train d'onde ». Un examen plus détaillé fait ressortir les concepts les plus pertinents : l'autocorrélation du champ et le bruit classique de détection.

Article publié en accès libre sous les conditions définies par la licence Creative Commons Attribution License CC-BY ( ), qui autorise

sans restrictions l'utilisation, la diffusion, et la reproduction sur quelque support que ce soit, sous réserve de citation correcte de la publication originale.

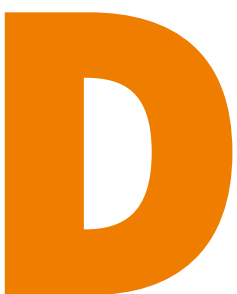

ans le domaine optique, les fréquences du champ électromagnétique se mesurent en centaines de térahertz. Les détecteurs ne peuvent pas suivre le champ électromagnétique : ils sont sensibles à son carré, intégré sur leur temps de mesure et sur leur aire sensible. Appelons « éclairement » cette grandeur, rapportée à l'unité d'aire, qui est donc l'intégrale sur une durée de mesure propre au détecteur de « l'éclairement instantané » et considérons une situation où la polarisation n'intervient pas. Le phénomène d'interférence illustre ce comportement quadratique : lorsqu'un détecteur reçoit de la lumière par deux voies différentes fournissant respectivement des champs électromagnétiques $E_{1}$ et $E_{2}$, le carré de la somme des champs ne se ramène pas à la somme des carrés des champs, léclairement n'est donc pas égal à la somme des éclairements produits par les deux voies : l'existence des interférences traduit le fait élémentaire que $\mathrm{E}^{2}=\left(E_{1}+E_{2}\right)^{2}$ diffère de $E_{1}^{2}+E_{2}^{2}$ ! Et pourtant, l'expérience quotidienne montre que son observation requiert des précautions : il faut s'assurer de la « cohérence » des champs $E_{1}$ et $E_{2}$. Nous examinons ici les conditions nécessaires pour obtenir cette cohérence. Autrement dit, pourquoi, sans précaution particulière, le terme croisé $\mathrm{E}_{1} \mathrm{E}_{2}$ du développement du carré est-il de moyenne nulle sur le temps d'intégration du récepteur?

La cohérence est ainsi en premier lieu une question temporelle. Nous consacrons ces lignes à la cohérence temporelle, les aspects spatial et vectoriel (polarisation) méritant un traitement séparé.

\section{UN MODĖLE SIMPLE L'ÉLECTRON ÉLASTIQUEMENT LIÉ}

Partant d'une représentation à la fois intuitive et instructive de la cohérence temporelle, revenons au modèle bien connu de lélectron élastiquement lié, qui présente en outre l'avantage d'une analogie étroite avec la physique d'un des systèmes quantiques les plus simples : l'atome à deux niveaux relaxant de son niveau supérieur vers son niveau inférieur, stable, en émettant spontanément un photon. Tout en restant ici dans le cadre de la physique classique, retenons que l'énergie élémentaire mise en jeu lors de l'émission et de la détection de lumière est celle d'un photon, extrêmement faible à notre échelle : les détecteurs sont le plus souvent sensibles à l'accumulation d'un grand nombre de photons. Dans une deuxième étape, nous prendrons du recul pour envisager des situations moins simplistes.

L'encart 1 rappelle qu'un oscillateur mécanique élastiquement lié et amorti, initialement excité, retourne spontanément vers son état de repos par une vibration sinusoïdale exponentiellement amortie, représentée exponentiellement amortie, ••• 
représentée à gauche de la figure de l'encart. Son spectre, le carré de sa transformée de Fourier, a une forme caractéristique dite lorentzienne (figure de droite). Si cet oscillateur modélise un électron autour d'un noyau atomique, il relaxe en émettant le champ électromagnétique d'une onde lumineuse qui suit la même loi temporelle, couramment appelé «train d'onde ».

Envoyons cette onde sur un interféromètre schématisé sur la figure 1 par deux miroirs dont les réglages permettent de modifier séparément les deux longueurs de trajet entre la source, considérée comme ponctuelle, et le détecteur. Les interférences sont observées en un point $M$ où se superposent les champs $E_{1}$ et $\mathrm{E}_{2}$ des deux voies. Considérons pour simplifier que l'indice du milieu est unitaire et que les deux éclairements créés par ces champs sont égaux. Les

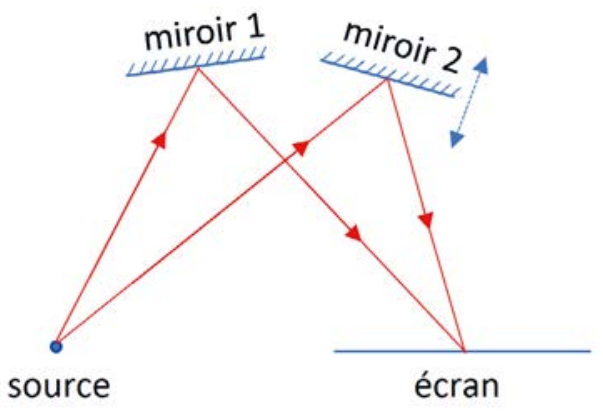

Figure $\mathbf{1}$ : un schéma simpliste d'interféromètre: deux parties du faisceau émis par la source interfèrent sur l'écran après s'être réfléchis sur deux miroirs de positions réglables.

champs $E_{1}$ et $E_{2}$ ne diffèrent alors que par le temps de propagation : $E_{2}$ est en retard (algébrique) d'une durée $\tau$ par rapport à $E_{1}$. Or, on l'a dit, et c'est crucial, la détection ne fait pas intervenir un seul photon, mais un grand nombre de photons. Lors de l'émission spontanée, que ce soit par un seul atome ou par une collection d'atomes très proches les uns des autres, l'instant d'émission d'un photon est indépendant de tous les autres. Parlant d'émission spontanée, nous excluons ici le laser (voir encart 2). Dès lors, les champs $E_{1}$ et $E_{2}$ ne doivent pas être conçus comme une seule émission semblable à celle de la figure 1, mais un grand nombre de telles émissions à des instants aléatoires indépendants les uns des autres.

L'interprétation élémentaire, et fondamentalement juste bien que schématique, de la cohérence temporelle est que si les deux répliques d'un train d'onde donné se chevauchent au point $M$, les interférences peuvent avoir lieu. Si cependant la différence de temps de trajet $|\tau|$ entre les deux voies de l'interféromètre est largement supérieure à la durée $\theta$ du train d'onde (mesurée ici 1/e du maximum initial), il ne peut interférer qu'avec

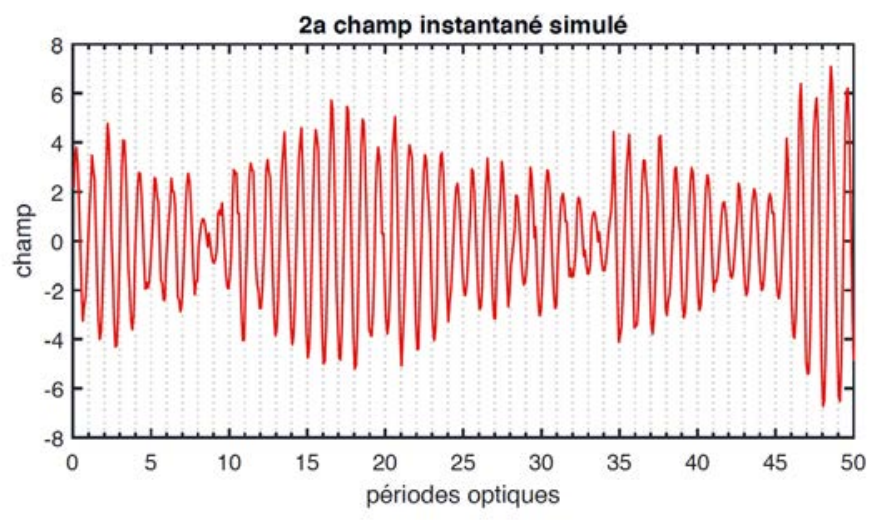

Figure 2 : simulation de la superposition d'un grand nombre de trains d'onde de même forme que la figure de l'encart, mais émises à des instants aléatoires indépendants à la cadence de 2 trains d'onde par période moyenne $T_{\text {o, }}$ en amplitude (a), en éclairement instantané (b) et en situation d'interférences pour trois valeurs du délai temporel $\tau$ (c).
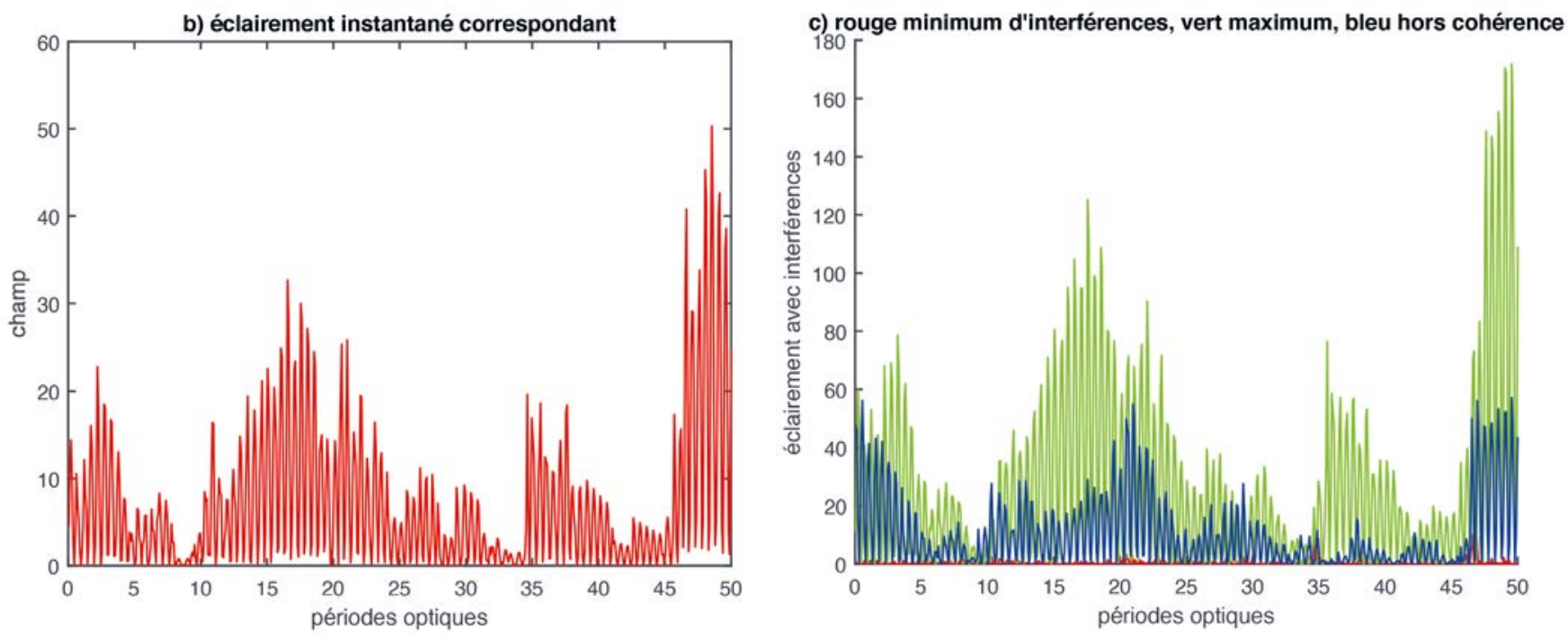


\section{ENCART 1 - L'OSCILLATEUR MÉCANIQUE ÉLASTIQUEMENT LIÉ}

On connaît le modèle du mouvement de la masse $m$ suspendu à un ressort de raideur $\mathrm{k}$ et de coefficient d'amortissement a, régi par l'équation différentielle $m d^{2} x / d t^{2}+a d x / d t+k x=0$, où $x$ est l'abscisse du centre de masse à partir de sa position de repos. La solution est une exponentielle dont la pulsation complexe $\omega$ est celle des deux solutions de l'équation $m \omega^{2}-\mathrm{i} a \omega-k=0$ qui assure un amortissement et non une amplification.
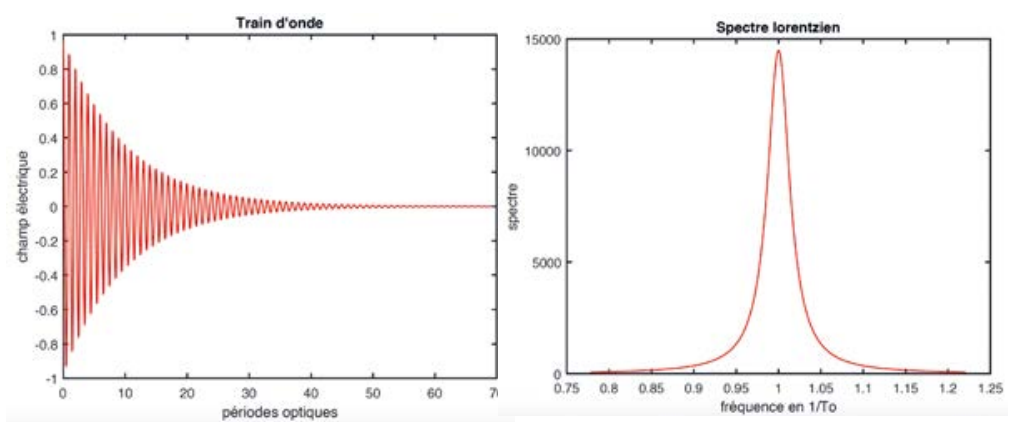

À gauche, l'allure de la loi temporelle $x(t)=x_{0} \cos \left(2 \pi t / T_{0}+\varphi\right) \exp (-t / \theta)$ et, à droite, son spectre lorentzien, carré du module de sa transformée de Fourier du côté des fréquences positives. Les constantes $T_{\mathrm{o}}$ et $\theta$ se déduisent des coefficients de l'équation différentielle et les constantes $x_{0}$ et $\phi$ traduisent les conditions initiales. La largeur du spectre est $1 / \pi \theta$. Dans le modèle de l'électron élastiquement lié, le mouvement de l'électron obéit à la même équation que celle de ce ressort.

d'autres trains d'onde qui fortuitement arrivent à ce même moment, mais non synchronisés, et par intégration sur le temps de détection de tous les trains d'onde concomitants présentant des déphasages aléatoires et indépendants, on n'observe pas d'interférence, mais un bruit, dit « bruit classique de détection ».

\section{SIMULATION D'UNE VIBRATION LUMINEUSE ET \\ DE SES INTERFÉRENCES}

Pour les lecteurs de Photoniques, il convient de montrer en quoi le modèle du train d'onde de l'électron élastiquement lié donne une image trop particulière de la réalité.

Pour cela, la figure 2a simule la superposition d'un grand nombre $N$ de trains d'onde sur le temps d'intégration $\Theta \mathrm{du}$ récepteur, long devant la durée $\theta$ du train d'onde et afortiori devant la période $T_{\text {o }}$ correspondant au centre du spectre ; les rapports entre les paramètres $\Theta, \theta$, et To ne sont pas réalistes mais ont été adaptés aux besoins de l'illustration. En général, $\Theta$ est beaucoup plus long. Par rapport à la figure de l'encart, on retrouve le caractère oscillant de la vibration élémentaire initiale, avec la même période moyenne $T_{0}$, mais deux caractéristiques nouvelles apparaissent : d'une part, l'amplitude de vibration instantanée varie dans de grandes proportions avec le temps, à une échelle temporelle semblable à celle de la durée $\theta$ des trains d'onde. La grille de période $T_{0}$ fait d'autre part apparaître les variations aléatoires de la phase instantanée à cette même échelle temporelle. La figure $2 b$ présente l'éclairement instantané $E^{2}$ correspondant. Rappelons que le détecteur est sensible à l'intégrale de cette dernière quantité sur la durée $\Theta$.

Considérons maintenant, en figure $2 \mathrm{c}$, les interférences entre deux ondes suivant ce même motif temporel. À la vibration initiale, représentant le champ $\mathrm{E}_{1}$, a été ajouté le champ $\mathrm{E}_{2}$ dans le cas d'un retard égal respectivement à $\tau=\mathrm{T}_{\mathrm{o}} / 2$, puis $\mathrm{T}_{\mathrm{o}}$ et $4 \theta$. On visualise par des $\bullet \bullet$

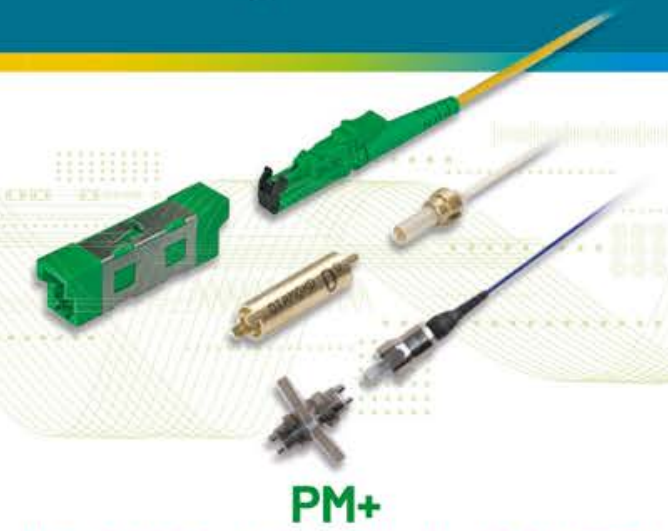

Ultra high Polarization Extinction Ratio (PER)

- Up to $+4 \mathrm{~dB}$ higher PER

- State-of-the-art insertion Loss (IL) and Return Loss (RL) values

- Best connector type and tolerance $\mathrm{E}-2000^{\circ}$, DMI, Mini $\mathrm{AVIM}^{\circ}$ and Micro $\mathrm{AVIM}^{\circ}$

- Available on homologated fibres and cables
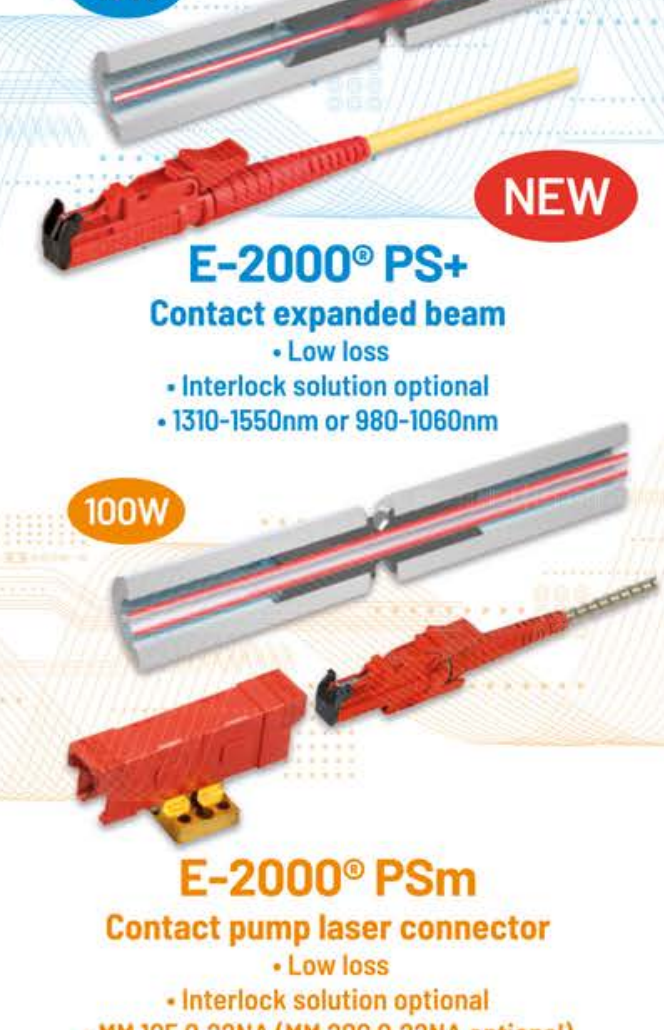

- MM 105 0.22NA (MM 200 0.22NA optional)

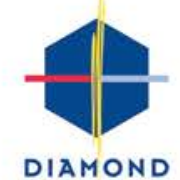

www.2blighting.fr

info@2blighting.com

+33164592130 


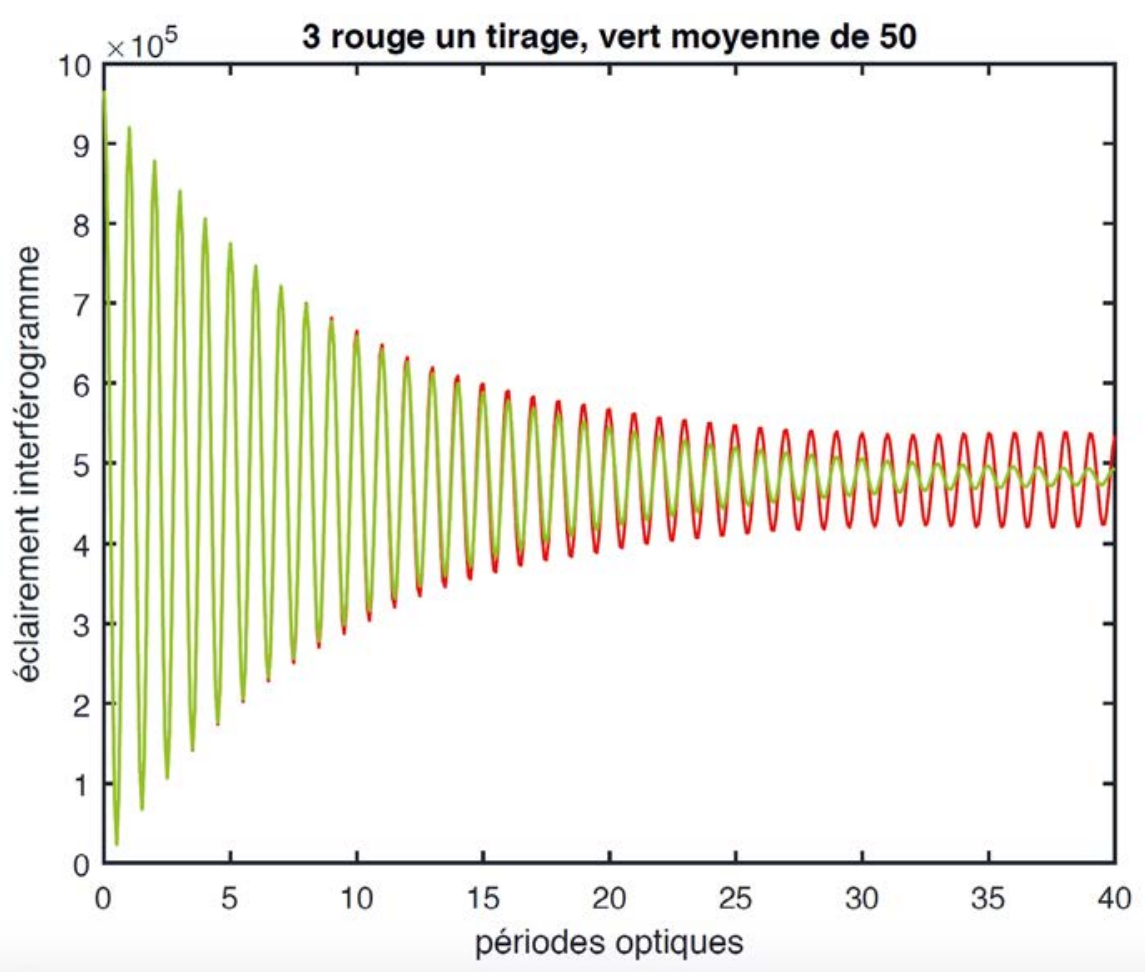

Figure 3 : simulation de la figure d'interférence en fonction du retard $\tau$ entre les deux vibrations pour $\Theta=40 \theta$. Le bruit classique de détection est révélé par la différence, en dehors des faibles valeurs de $\mathrm{\tau}$, entre l'accumulation de 50 tirages indépendants des instants d'émission des trains d'onde (en vert) et un tirage unique (courbe en rouge, initialement masquée par la courbe verte).

couleurs différentes les éclairements instantanés correspondants : respectivement, un minimum d'interférences, un maximum, et le double de l'éclairement de la figure $2 \mathrm{~b}$. Autrement dit, dans les deux premiers cas, le produit croisé $\mathrm{E}_{1} \mathrm{E}_{2}$ se traduit par des interférences, et dans le troisième, il a une moyenne nulle, les interférences ne se produisent pas : les deux vibrations, bien qu'identiques en forme, décalées d'un temps trop long, ne sont pas cohérentes. La figure 3, enfin, est la simulation d'un interférogramme, représenté entre 0 et $\Theta$ en fonction du temps de retard $\tau$. On voit que

Figure 4 : interférogrammes de Michelson obtenus (de gauche à droite) avec une lumière blanche, la même passée à travers un filtre interférentiel vert, et le spectre de raies d'une lampe à mercure. la « modulation » locale calculée sur une pseudo-période $T_{0}$ de l'interférogramme $\left(E_{\max }-E_{\min }\right) /\left(E_{\max }+E_{\min }\right)$ décroit avec un temps caractéristique $\theta$ selon l'exponentielle de train d'onde initial. Lorsque le retard $\tau$ du trajet 2 sur le trajet 1 est nettement plus grand que ce temps caractéristique $\theta$, la cohérence est perdue. $\theta$ porte pour cette raison le nom de «temps de cohérence ». Notons au passage que la distance $c \theta$ que parcourt la lumière pendant la durée $\theta$, bien qu'appelée «longueur de cohérence », décrit bien la cohérence temporelle et non pas la cohérence spatiale.

\section{AUTOCORRÉLATION ET SPECTRE}

Revenons pour terminer sur la relation entre la figure d'interférence observée et les variations temporelles du champ électrique qui résulte de tous les évènements aléatoires élémentaires indépendants. L'intégrale du carré du champ s'étend sur la durée d'intégration $\Theta$ du détecteur. La figure 3 illustre la nécessité d'un long temps d'intégration (ou, ce qui revient pratiquement au même, d'une accumulation statistique de tirages indépendants) pour que la figure d'interférences soit libérée des perturbations liées au caractère aléatoire du champ instantané. Ces fluctuations, appelées «bruit classique de détection », deviennent imperceptibles dans le cas pratique ordinaire où $\theta$ est très brève par rapport à $\Theta$ : c'est alors que les interférences apparaissent pleinement, dévoilant l'intégrale sur le temps de détection du terme croisé $E_{1}(t) E_{2}(t)$. Ce produit est en fait l'intégrale de $E_{1}(t) E_{1}(t-\tau)$, ce qui revient à la fonction d'autocorrélation du champ électrique, considéré comme un processus aléatoire.

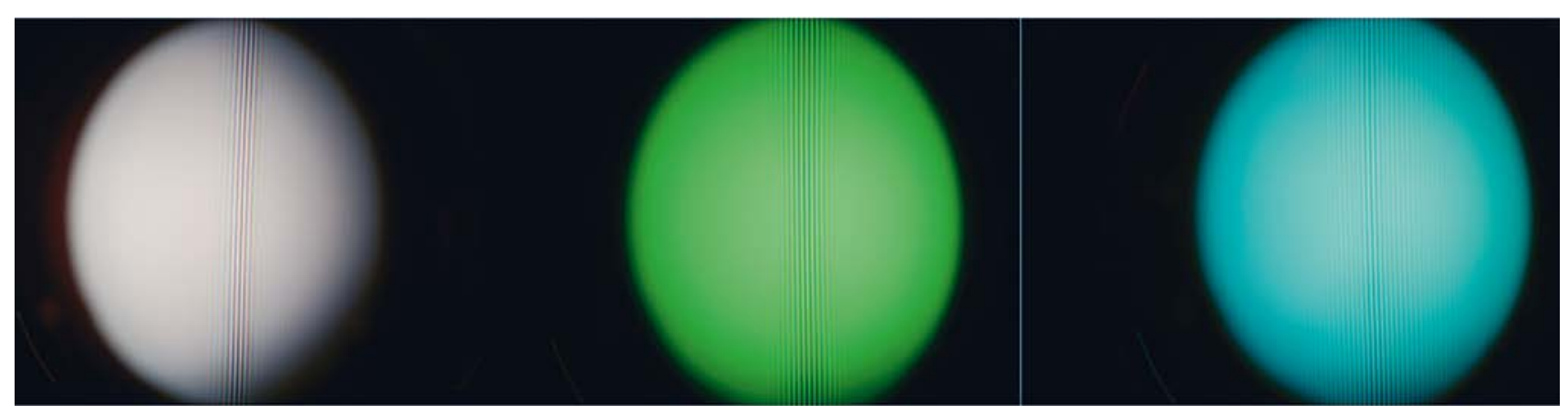




\section{ENCART 2 - LA COHÉRENCE TEMPORELLE DANS LES LASERS}

En raison de l'émission stimulée qui synchronise les phases de tous les photons, les propriétés temporelles des lasers diffèrent notablement de celles des "sources thermiques » dont traite cet article. Elles sont cependant diversifiées en raison de la multiplicité des régimes de fonctionnement des lasers, notamment en ce qui concerne leurs modes temporels. En régime monomodal temporel, la cohérence n'est limitée que par les résidus d'émission spontanée et par les inévitables dérives ou vibrations du montage; mais de nombreux lasers émettent simultanément sur plusieurs modes temporels. En régime continu ainsi qu'en mode relaxé ou en régime déclenché par le gain ou les pertes, de nombreux modes peuvent coexister et ne sont pas synchronisés ; on s'approche alors des statistiques et des propriétés de cohérence du régime thermique, à cette différence près que les allers-retours dans la cavité laser créent une cohérence temporelle périodique de période égale au temps d'allerretour. Au contraire, dans tous les régimes de verrouillage de modes, chaque cas est particulier. Si les impulsions peuvent être étudiées individuellement, la relation de phase entre modes et donc la structure temporelle peut être fixe ou bien aléatoirement variable d'une impulsion à l'autre mais observable. Pour les impulsions ultrabrèves comme pour les peignes de fréquence au contraire, une synchronisation très stricte de tous les modes est essentielle; diverses lois de phase peuvent d'ailleurs se présenter. On voit que dans tous ces derniers cas, la notion de cohérence temporelle n'a pas la même pertinence qu'en régime "thermique » stationnaire.

Or, dans le cadre mathématique approprié, l'analyse harmonique des processus aléatoires stationnaires, le théorème de Wiener-Khintchine établit que cette fonction d'autocorrélation a pour transformée de Fourier le spectre de la source. Par transformée de Fourier de l'interférogramme, on obtient le spectre de la source : c'est le principe de la spectrométrie de Fourier, et la loi de base qui régit la cohérence temporelle. Il en résulte aussi que les interférences ne donnent pas d'autre information sur la source que son spectre ; autrement dit, les interférences ne disent rien sur les trains d'onde : une infinité de fonctions ont la même autocorrélation, et donc le même spectre. C'est seulement un modèle physique sous-jacent à l'émission de lumière par des milieux dilués qui peut permettre d'introduire les trains d'onde, ainsi que leur modification par collisions ou effet Doppler, leur multiplicité dans un spectre de raies, et de construire alors un modèle de spectre qui peut être comparé aux données expérimentales de l'interférométrie. Si la lumière est émise par un solide, le concept de train d'onde présente beaucoup moins d'intérêt.

La figure 4 résume ces propriétés en juxtaposant trois interférogrammes d'égale épaisseur obtenus à l'interféromètre de Michelson : le nombre des franges visibles croît de gauche à droite, indiquant un rétrécissement du spectre, et le profil de leur modulation renseigne en détail sur ce dernier.

\section{VOTRE PARTENAIRE : Photométrie Radiométrie \& Colorimétrie}

\section{Radiomètres UV-C}

Désinfection

Contrôle de source UV (LED UV, Excimer \& Mercure basse pression) Polymérisation

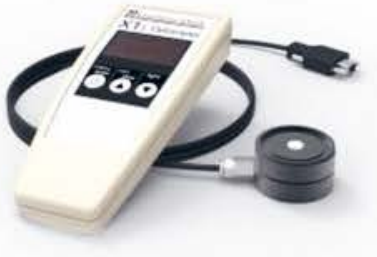

Colorimètres \& Vidéocolorimètres

Mesures de l'uniformité en couleur ou luminance de vos sources ou displays

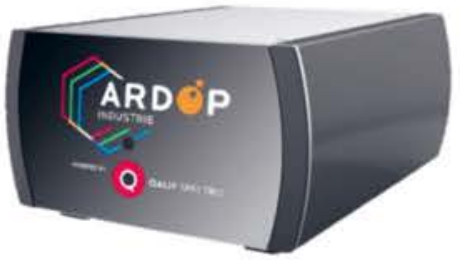

\section{Nouveau Spectroradiomètre IR}

\section{Technologie BiTec : détecteur à matrice InGaAs + diode InGaAs \\ Refroidissement thermoélectrique} Plage spectrale $950-2150 \mathrm{~nm}$

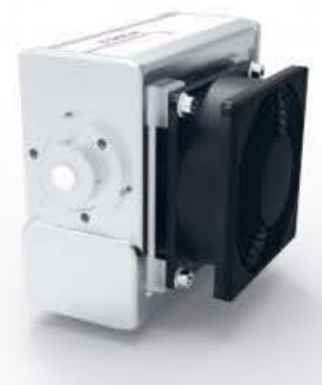

